\title{
Características clínicas básicas en los primeros 100 casos fatales de COVID-19 en Colombia
}

\author{
Alejandro Marín-Sánchez ${ }^{1}$
}

Forma de citar Marín-Sánchez A. Características clínicas básicas en los primeros 100 casos fatales de COVID-19 en Colombia. Rev Panam Salud Publica. 2020;44:e87. https://doi.org/10.26633/RPSP.2020.87

RESUMEN

Se analizaron las características clínicas básicas de los primeros 100 casos de fallecimientos por COVID-19 en Colombia con base en los reportes del Instituto Nacional de Salud (INS) desde el inicio de la pandemia. Dado que el registro del INS no toma en cuenta las variables clínicas de comorbilidad en el total de los casos reportados como positivos, sino solo en los pacientes con desenlace mortal, se revisaron la patología concomitante, la edad y el sexo disponibles en los reportes diarios del INS. Se identificó su frecuencia y se intentó establecer el comportamiento de riesgo de mortalidad para las variables analizables (OR), y se comparó con el comportamiento descrito en la literatura internacional. De los 100 casos, 63 fueron de sexo masculino, el promedio de edad fue 65,75 \pm 18,11 años y en 22 de ellos no se había reportado comorbilidad. Las patologías informadas con más frecuencia fueron hipertensión arterial (35\%), diabetes mellitus (21\%), enfermedades cardiovasculares y cerebrovasculares (19\%), enfermedad pulmonar obstructiva crónica (16\%), obesidad (12\%), tabaquismo (9\%) y patología tiroidea (8\%). Los pacientes mayores de 60 años presentaron un mayor riesgo de mortalidad (OR 10,31, IC95\% 6,67-15,94, p < 0,0001). El 10\% de los pacientes fallecidos eran menores de 60 años y no presentaban comorbilidad.

Aunque los esfuerzos por parte de los entes gubernamentales han sido grandes, en materia de la información del curso de la infección por COVID-19 en la población colombiana aún no se ha dado a conocer un reporte o un análisis de las características clínicas, en especial la comorbilidad, de los pacientes afectados. Las condiciones mórbidas existentes solo se han reportado en los casos con desenlace fatal, pero estas no se han informado en los supervivientes. Ante la descripción en la literatura de factores de riesgo relacionados con enfermedades endócrinas y cardiovasculares se llevó a cabo un análisis preliminar de la información disponible.

\section{MATERIALES Y MÉTODOS}

Se tomó como referencia el reporte diario de los pacientes con confirmación de infección por COVID-19 por laboratorio (centralizado a nivel nacional en el Instituto Nacional de Salud [INS] al momento del análisis), desde la confirmación del primer caso en Colombia el 6 de marzo de 2020 (correspondiente a una paciente de 19 años procedente de Milán, Italia) hasta el 11 de abril de 2020, día en que se alcanzó una mortalidad de 100 casos confirmados en el país. (1) Los datos considerados en los reportes diarios del INS solo incluyen distribución geográfica, edad, sexo, tipo de caso (importado o relacionado), país de origen y fechas de diagnóstico, muerte y recuperación para todos los casos confirmados positivos de COVID-19. La comorbilidad es registrada en la base de datos solo en los caso con desenlace fatal. Se realizó la tabulación de los datos clínicos reportados día a día por el INS con el fin de determinar la frecuencia de presentación de la comorbilidad y una posible definición de riesgo de mortalidad (OR) según las variables analizables, y comparar el comportamiento de estas con lo descrito en los reportes internacionales.

\footnotetext{
1 Universidad de Caldas, Manizales, Colombia $₫$ Alejandro Marín-Sánchez, lexmarcorp@hotmail.com
} 


\section{RESULTADOS}

De acuerdo al origen, solo 7 de los casos han sido establecidos como importados, 34 como relacionados por nexo epidemiológico y 59 se encuentran aún en estudio.

La distribución geográfica de los casos mostró que la mayor frecuencia se presentó en las ciudades de Bogotá ( 45 casos), Cali (10 casos) y Cartagena (6 casos). En la evaluación por regiones, en la Costa Atlántica se presentaron 11 casos (Santa Marta 4, Barranquilla 2, Soledad 2, Montería 1, Ciénaga de Oro 1, Turbaco 1); en el Eje Cafetero, Antioquia y Huila 9 casos (Neiva 3, La Dorada 2, Pereira 2, Armenia 1, Medellín 1); y en la región cundiboyacense y los llanos orientales se han reportado 9 casos (Villavicencio 3, Villapinzón 2, Tunja 1, Zipaquirá 1, Tenjo 1, Suesca 1). Por último, en la región de los Santanderes se observaron 4 casos (Cúcuta 2, Barrancabermeja 1, Ocaña 1) y en los departamentos del sur del país 6 casos (Pasto 2, Ipiales 2, Popayán 1, Santander de Quilichao 1). En el cuadro 1 se presentan las características principales de los pacientes. En 22 de los casos no se identificó morbilidad asociada, y 10 de ellos correspondían a pacientes menores de 60 años. La comorbilidad asociada a disglucemia se identificó como diabetes mellitus de tipo 2 (DM2), pero no era claro en todos los casos el grado de control de la glucemia.

La mortalidad ocurrió en el rango de edad desde los 3 hasta los 98 años, llamando la atención en dichos valores extremos la presencia de síndrome de Alagille y la ausencia de comorbilidad reportada en el paciente casi-centenario. El promedio de edad de los pacientes fallecidos $(65,75 \pm 18,11)$ con comparación con los supervivientes $(43,3 \pm 18,03$ años) presentó una diferencia significativa (diferencia absoluta 22,4 años, IC95\% 18,84-25,05, $\mathrm{p}<0,0001)$.

En la categoría de enfermedad cardiovascular el $6 \%$ de los casos se informaron como enfermedad cardíaca, sin especificación del origen de esta. La comorbilidad reportada con mayor frecuencia fue la hipertensión arterial, que en 10 casos se presentó junto a diabetes mellitus, en 6 casos se asoció con obesidad y en 2 con dislipidemia. La hipertensión se asoció con compromiso cardiovascular como falla cardíaca, enfermedad cardíaca no especificada, enfermedad coronaria y enfermedad renal crónica en 2 casos cada una y con enfermedad cerebrovascular, cardiopatía hipertensiva, angioplastia y aneurisma cerebral en un caso cada una. Respecto de la diabetes, se reportó asociación con obesidad en 2 casos, en uno con dislipidemia, cardiopatía hipertensiva y falla cardíaca en un caso cada una, y enfermedad cerebrovascular en 2 casos.

Se reportaron algunos antecedentes quirúrgicos, pero solo en un caso hubo proximidad temporal con la infección (un paciente masculino procedente de Ecuador, tras una cistostomía suprapúbica). En otros 2 casos se había reportado como antecedente histerectomía y safenectomía, pero sin precisión respecto del momento de las intervenciones.

Aunque el promedio entre el diagnóstico de la infección y el fallecimiento fue cercano a 3 días (promedio 3,35 días $\pm 4,3$ días, rango 0-18 días), en el 33\% de los casos la fecha del diagnóstico de laboratorio era el mismo día del reporte del fallecimiento. Sólo en 10 casos hubo una diferencia entre el diagnóstico y el fallecimiento mayor de 10 días (rango 10-18 días).

Al momento en que se reportó el caso fatal 100, solo se había informado un caso fatal en personal de salud (médico de 33 años con hipertiroidismo de base). Posteriormente se informó
CUADRO 1. Características básicas 100 primeros pacientes fallecidos por COVID-19 Colombia

\begin{tabular}{|c|c|}
\hline Característica & Frecuencia \\
\hline \multicolumn{2}{|l|}{ Sexo } \\
\hline Masculino & 63 \\
\hline Femenino & 37 \\
\hline \multicolumn{2}{|l|}{ Edad } \\
\hline Media $\pm D E$ & $65,75 \pm 18,11$ \\
\hline Rango & $3-98$ \\
\hline Moda & 72 \\
\hline Mediana & 70 \\
\hline Menores de 60 años & 31 \\
\hline \multicolumn{2}{|l|}{ Comorbilidad } \\
\hline No reportada & 22 \\
\hline Hipertensión arterial & 35 \\
\hline Diabetes Mellitus & 21 \\
\hline Enfermedad pulmonar obstructiva crónica & 16 \\
\hline Obesidad & 12 \\
\hline Tabaquismo & 9 \\
\hline Patología tiroidea ${ }^{a}$ & 8 \\
\hline Enfermedad renal crónica ${ }^{b}$ & 5 \\
\hline Dislipidemia & 4 \\
\hline \multicolumn{2}{|l|}{ Patología pulmonar adicional } \\
\hline Asma & 2 \\
\hline Tromboembolismo pulmonar & 1 \\
\hline Hipertensión pulmonar & 1 \\
\hline \multicolumn{2}{|l|}{ Patología cardiovascular o cerebrovascular } \\
\hline Enfermedad cardíaca & 6 \\
\hline Enfermedad coronaria $^{c}$ & 3 \\
\hline Falla cardíaca & 2 \\
\hline Arritmia supraventricular & 2 \\
\hline Angina inestable & 1 \\
\hline Fibrilación auricular & 1 \\
\hline Cardiopatía hipertensiva & 1 \\
\hline Evento cerebrovascular & 3 \\
\hline \multicolumn{2}{|l|}{ Compromiso neurológico } \\
\hline Epilepsia & 1 \\
\hline Aneurisma cerebral & 1 \\
\hline Demencia $^{d}$ & 2 \\
\hline Secuelas de hipoxia cerebral & 1 \\
\hline Enfermedad de Parkinson & 1 \\
\hline Neoplasias ${ }^{e}$ & 4 \\
\hline Compromiso reumatólógico ${ }^{\dagger}$ & 2 \\
\hline Sindrome de Prader-Willi & 1 \\
\hline Sindrome de Allagile & 1 \\
\hline
\end{tabular}

1 paciente con reporte de hipertiroidismo; los restantes, hipotiroidismo

c 1 paciente con reporte de coronariografía y stent por enfermedad de 2 vasos en 2009 y 1 paciente con infarto de miocardio en 2003

• Cáncer de mama con diagnóstico 19 años antes 1, cáncer de pulmón 1, mieloma múltiple 1, tumor en la vía biliar (malignidad no aclarada)

1 paciente lupus eritematoso, 1 paciente artritis reumatoidea

un segundo caso (médico varón de 57 años, no incluido oficialmente antes del cierre del reporte diario del 11 de abril de 2020.

\section{DISCUSIÓN}

Al 11 de Abril de 2020, según el reporte oficial, se habían confirmado 2709 casos de COVID-19 en Colombia, con 214 
pacientes recuperados y 100 casos fallecidos. Algunas dificultades en la disponibilización de las pruebas en la población y la centralización del procesamiento han ocasionado demoras en la identificación de casos positivos, incluso en los casos fatales. Respecto del comportamiento clínico, actualmente resulta preocupante que el $10 \%$ de los pacientes fallecidos eran menores de 60 años, sin morbilidad reportada. Sin embargo, cabe la posibilidad de subregistro en el proceso inicial de recolección en las fichas epidemiológicas durante el trabajo de campo para el reporte oficial, pues en el 12\% de los pacientes mayores de 60 años tampoco se informaban patologías en un rango etario que se extendía casi hasta los 100 años. Por las condiciones evidenciadas de síndrome metabólico, enfermedad cardiovascular y enfermedad renal en nuestro medio cabría esperar una presencia más elevada de hipertensión arterial, diabetes mellitus de tipo 2 o disglucemia, dislipidemia y obesidad en forma simultánea en un mismo paciente, lo cual podría deberse también a subregistro.(2-6) Aun así, llama la atención la frecuencia de diabetes, muy por encima de lo reportado en otras series de casos asociados a mortalidad por COVID-19 y de la prevalencia en nuestra población general (entre el 9 y 10\% de los casos). (7-8) En relación con la enfermedad renal crónica, en un caso se asoció con nefropatía diabética, en otro paciente con diabetes mellitus de tipo 2 y en dos casos con hipertensión arterial. De manera similar, una limitación importante en el registro es la falta de discriminación del registro de enfermedad cardíaca con asignación de alguna etiología específica.

Estudios de cohorte retrospectivos y metaanálisis específicos han demostrado la asociación de mortalidad con múltiples factores de riesgo, siendo los principales hipertensión arterial $(30 \%)$, diabetes $(19 \%)$, enfermedad arterial coronaria $(8 \%)$ y enfermedad pulmonar crónica obstructiva (7\%).(2) Un metaanálisis en particular enfocado en la hipertensión arterial, que incluyó 13 estudios (3), demostró un incremento de 2,5 veces de enfermedad severa por COVID-19 (OR 2,49, IC95\% 1,983,12 ) con un riesgo de mortalidad similar (OR 2,42, IC95\% 1,51-3,9) y una correlación significativa con la edad. Con dichas frecuencias se han establecido incrementos de riesgo importantes para características clínicas -no necesariamente patológicas-, como la mayor edad (OR 1,10, IC95\% 1,03-1,1; $\mathrm{p}=0,0043$; promedio 69 frente a 52 años; $\mathrm{p}<0,001)$. En algunos países, como el Reino Unido, se ha considerado que hasta un $25 \%$ de la población estaría expuesta a un alto riesgo debido a su edad y comorbilidad.(4) De igual forma, algunas series de casos han demostrado que la obesidad y el tabaquismo tuvieron impacto en el incremento del riesgo, y en países como Italia parece existir correlación con el sexo (mayor compromiso en varones), que se ha asociación con el tabaquismo.(4, 5) Con respecto al contexto metabólico y la obesidad, en un análisis retrospectivo de 389 pacientes se evidenció que 32\% de ellos presentaban sobrepeso y $10,7 \%$ obesidad, y tuvieron un riesgo
2,42 veces mayor de neumonía severa, sin diferencias significativas de sexo $(p=0,09)$. (6)

Aunque en la serie de casos estudiada la patología tiroidea tiene una representación importante, en la literatura internacional no hay una correlación clara de mayor riesgo de COVID-19. En un estudio llevado a cabo durante la epidemia de síndrome respiratorio agudo severo (SARS) en 2002 se evaluó la posibilidad de afectación viral del tejido tiroideo, y aunque se encontró un efecto apoptótico en el epitelio folicular que podría asociarse con tiroiditis, el tamaño de la muestra $(n=5)$ y la correlación clínica no permitieron definir un papel concreto del compromiso infeccioso sobre la función tiroidea.(9)

Al igual que en nuestro estudio, los estudios internacionales reportaron dificultades para controlar factores como la edad, el sexo y el tabaquismo (reportado en distintos estudios entre $7 \%$ y $>60 \%$ ), así como el tiempo de seguimiento, el registro incompleto o el inadecuado diagnóstico de las condiciones subyacentes y el sesgo de selección de una población con mayor riesgo. $(10,11)$

Al igual que en otras poblaciones (2) el sexo masculino presentó mayor mortalidad (63 frente a $37 \%$ ), pero con un incremento porcentual importante respecto de otras publicaciones con mortalidad también superior en varones (OR 3,4 IC95\% 1,2 a 9,1, p = 0,01).(12) Aun con las limitaciones existentes en la información de base, con los datos disponibles se evidenciaría un riesgo mayor (OR 1,7; IC95\% 1,13-2,58; $\mathrm{p}=0,0110$ ) para el sexo masculino. En los pacientes de más de 60 años el riesgo de mortalidad sería mayor en forma muy significativa (OR 10,31; IC95\% 6,67-15,94; p < 0,0001). Debido a la falta de información sobre comorbilidad en los pacientes sin desenlace fatal, no es posible estimar el riesgo por patologías.

Actualmente, la limitación principal para llevar a cabo un análisis más detallado de los factores asociados con la mortalidad por COVID-19 en Colombia es la falta de información complementaria paraclínica y de las variables clínicas específicas. El registro actual, centralizado con los datos de los pacientes, presenta un posible subregistro de la comorbilidad y se requerirá un registro y una revisión de las historias clínicas más detallados para complementar los datos de las características clínicas básicas y datos complementarios como las variables de la evolución y el manejo, el soporte ventilatorio y hemodinámico, marcadores de respuesta inflamatoria o bioquímicos, o la clínica inicial. Dicha información brindaría una mayor comprensión de los perfiles de riesgo, con posibles repercusiones en materia de salud pública para el país ante la pandemia.

Conflicto de intereses. Ninguno declarado por los autores.

Declaración. Las opiniones expresadas en este manuscrito son responsabilidad del autor y no reflejan necesariamente los criterios ni la política de la RPSP/PAJPH y/o de la OPS.

\section{REFERENCIAS}

1. Instituto Nacional de Salud Colombia. Coronavirus (COVID-19) en Colombia. [Internet] COVID-19 en cifras. (Citado 2020, Abr 11) Disponible en: https://www.ins.gov.co/Noticias/Paginas/ Coronavirus.aspx

2. Zhou F, Yu T, Du R, Fan G, Liu Y, Liu Z, Xian J, et al. Clinical course and risk factors for mortality of adult inpatients with COVID-19 in Wuhan,
China: a retrospective cohort study. Lancet. 2020 Mar 28;395(10229): 1054-1062. doi: 10.1016/S0140-6736(20)30566-3. Epub 2020 Mar 11.

3. Lippi G, Wong J, Henry BM. Hypertension and its severity or mortality in Coronavirus Disease 2019 (COVID-19): a pooled analysis. Pol Arch Intern Med. 2020 Mar 31. doi: 10.20452/pamw.15272. Epub ahead of print. 
4. Jordan RE, Adab P, Cheng KK. COVID-19: risk factors for severe disease and death. BMJ. 2020;368:m1198. doi: 10.1136/bmj.m1198.

5. Wang D, Hu B, Hu C, et al . Clinical characteristics of 138 hospitalized patients with 2019 Novel Coronavirus-Infected pneumonia in Wuhan, China. JAMA 2020;323(11):1061-9. 10.1001/jama.2020.1585

6. Cai $Q$ and Chen F, Luo F, et al. Obesity and COVID-19 Severity in a Designated Hospital in Shenzhen, China (3/13/2020). Disponible en: http://dx.doi.org/10.2139/ssrn.3556658

7. Aschner P. Epidemiología de la diabetes en Colombia. Av Diabetol. 2010;26:95-100

8. Vargas-Uricoechea H, Casas-Figueroa LÁ. An Epidemiologic Analysis of Diabetes in Colombia. Ann Glob Health. 2015;81(6):742-53

9. Wei L, Sun S, Xu Ch, Zhang J, Xu Y, et al. Pathology of the thyroid in severe acute respiratory syndrome. Hum Pathol. 2007;38(1):95-102.

10. Li D, Lv J, Liu F, et al . Hypertension burden and control in mainland China: Analysis of nationwide data 2003-2012. Int J Cardiol. 2015;184:637-44. 10.1016/j.ijcard.2015.03.045 25771229
11. Liu S, Zhang M, Yang L, et al . Prevalence and patterns of tobacco smoking among Chinese adult men and women: findings of the 2010 national smoking survey. J Epidemiol Commun H. 2017;71:154-61. 10.1136/jech-2016-207805 27660401

12. Nasiri NJ, Haddadi S, Tahvildari A, Farsi $Y$, Arbabi M et al. COVID-19 clinical characteristics, and sex-specific risk of mortality: Systematic Review and Meta-analysis. medRxiv. 2020. DOI: $10.1101 / 2020.03 .24 .20042903$

Manuscrito recibido el 13 de abril de 2020. Aceptado para publicación, tras revisión, el 3 de junio de 2020.

\section{Basic clinical characteristics in the first 100 fatal cases of COVID-19 in Colombia}

\section{ABSTRACT}

Palabras clave
The basic clinical characteristics of the first 100 fatal cases from COVID-19 in Colombia were analyzed based on reports from the National Institute of Health (INS) since the beginning of the pandemic. Since the INS records do not include clinical variables of comorbidity in the total number of cases reported as positive, but only in patients with fatal outcome, comorbidities, age and sex available in the daily INS reports were reviewed. Their frequency was identified and mortality risk behavior for the analyzed variables was established and compared with the behavior described in the international literature. Of the 100 cases, 63 were male, the mean age was $65.75 \pm 18.11$ years, and in 22 of them no comorbidity had been reported. The most frequently reported comorbidities were arterial hypertension (35\%), diabetes mellitus (21\%), cardiovascular and cerebrovascular disease (19\%), chronic obstructive pulmonary disease (16\%), obesity (12\%), smoking (9\%) and thyroid disease (8\%). Patients over 60 years of age presented a higher risk of mortality (OR 10.31, IC95\% $6.67-15.94, p<0.0001)$. Ten percent of the deceased patients were under 60 years of age and did not present comorbidity.

Coronavirus; mortality; Colombia. 